http://www.jfas.info

\title{
NARX-BASED BPSO MODELLING FOR TIME-VARYING STEAM TEMPERATURE OF STEAM DISTILLATION PILOT PLANT
}

\author{
N. Hambali, M. N. Taib, A. I. M. Yassin and M. H. F. Rahiman* \\ Faculty of Electrical Engineering, Universiti Teknologi MARA, 40450 Shah Alam, Selangor, \\ Malaysia
}

Published online: 05 October 2017

\begin{abstract}
This paper focuses on a nonlinear modelling for a time-varying process of steam temperature by employing a polynomial Nonlinear Auto-Regressive with Exogenous Input (NARX) structure based on Binary Particle Swarm Optimization (BPSO) algorithm. The system identification time-varying steam temperature data was collected from Steam Distillation Pilot Plant. Three models' criterion were implemented; Akaike Information Criterion, Model Descriptor Length (MDL) and Final Prediction Error (FPE) for optimization process of NARX-based BPSO modelling. The results demonstrated that the FPE criterion model was presented a slightly better model with lowest CRV from the testing set, small fitness value and a minimum number of parameter in the output model. The accuracy was evaluated by the high R-squared, small MSE value and passed all the correlation and histogram tests.
\end{abstract}

Keywords: identification; distillation column; temperature; NARX; particle swarm optimization.

Author Correspondence, e-mail: hezrif@ieee.org

doi: http://dx.doi.org/10.4314/jfas.v9i4s.6 


\section{INTRODUCTION}

A system identification involves linear and nonlinear modelling [1-3]. A distillation column has reported by a broad application of the nonlinear dynamic behaviour [4-8].

Unfortunately, a linear modelling for the nonlinear system demonstrated some limitation due to the robustness and accuracy of the system [9]. Moreover, greater presentation and potentiality for the nonlinear model was obtained compared to lesser correctness and inadequate fit resulted in linear model modelling for nonlinear system representation [10-11]. The adequacy of nonlinear dynamic behaviours of the nonlinear system is demonstrated substantially and can be simply applied to control design. Furthermore, a time-varying and nonlinearity behaviour of the real system such as distillation column has been a serious matter in such cases like loss detection which most existing formulated models have a limitation in identifying this physical progression [12-13].

However, there is still a need for time-varying NARX modelling for a distillation column [13-15]. Furthermore, Binary Particle Swarm Optimization (BPSO) algorithm has not been practiced for the mentioned distillation column using polynomial time-varying NARX model $[16-17,28]$.

Time-varying dynamic behaviour is a progressively vital area in nonlinear system identification. Previous studies have magnificently developed the nonlinear and time-varying NARX model for semisubmersible platform, dielectric elastomer actuators and human EEG data $[13-14,18]$. Recently, time-varying modelling has proven the strength and accuracy finding of the nonlinearity process. These highly nonlinear responses were revealed through the accurate nonlinear model by selecting the significant terms in the model.

The structure selection aim is model parsimony which it should capable of clarifying the data dynamics by means of the last regressor terms number [19]. Model parsimony aims the best model with the to the lowest degree complexity between multiple model structures. Information criteria such as AIC, FPE and MDL are used to impose parsimony by integrating difficulty drawbacks in accession to residual fit [20-21]. Not only better precision but also skilful in the model fit enhancement and correlation violations (CRV) number decline were reported in the proposed BPSO method [22-24]. Additionally, for structure selection 
optimization, BPSO needed the least iterations with well fitness values for convergence [25]. NARX model with BPSO offered a reliable model fit for essential oil identification and appropriately concealed the dynamics of the time invariant system [26]. Subsequently, the previous study also presented optimal swarm size for convergence using BPSO-based NARX for DC Motor [27].

The purpose of this study is to presents a time-varying polynomial NARX for steam temperature by utilizing a BPSO algorithm for SDPP using PRBS input signal. AIC, FPE and MDL were employed for a fitness assessment.

\section{METHODOLOGY}

The NARX model:

$y(t)=f^{d}\left[\left(\begin{array}{c}y(t-1), y(t-2), \ldots, y\left(t-n_{y}\right), \\ u\left(t-n_{k}\right), u\left(t-n_{k}-1\right), \ldots, u\left(t-n_{k}-n_{u}\right)\end{array}\right)\right]+\varepsilon(t)$

The output $y(t)$, the input $u(t)$ and $n_{y}$ and $n_{u}$ are their corresponding maximum lags with the estimated model $\mathrm{f}^{\mathrm{d}}$. Typical value 1 is set for the input signal time delay $\mathrm{n}_{\mathrm{k}}$.

\subsection{Polynomial NARX}

NARX polynomial model:

$\mathrm{y}(\mathrm{t})=\sum_{\mathrm{m}=1}^{\mathrm{n}_{\mathrm{p}}} \mathrm{P}_{\mathrm{m}} \theta_{\mathrm{m}}+\varepsilon(\mathrm{t})$

$P_{1}=1$ for the $m$-th regression $P_{m}$ term, the $m$-th regression parameter $\theta_{m}$ and the number of terms in the polynomial expansion $\mathrm{n}_{\mathrm{p}}$.

The Least Squares (LS) formulation:

$\mathrm{P} \theta+\varepsilon=\mathrm{y}$

\subsection{Model Structure Selection: Binary Particle Swarm Optimization (BPSO)}

The Vanilla PSO algorithm:

$V_{\text {id }}=V_{\text {id }}+C_{1}\left(P_{\text {best }}-X_{i d}\right) \times \operatorname{rand}_{1}+C_{2}\left(G_{\text {best }}-X_{\text {id }}\right) \times \operatorname{rand}_{2}$

The velocity $V_{\mathrm{id}}$ has been employed for a particle positions alteration:

$X_{\text {id }}=X_{\text {id }}+V_{\text {id }}$

where $\mathrm{P}_{\text {best }}=$ best particle's fitness, $\mathrm{G}_{\text {best }}=$ best particle's solution, $\mathrm{C}_{1}=$ cognition learning rate, $C_{2}=$ social learning rate and $\operatorname{rand}_{1}, \operatorname{rand}_{2}=$ random numbers (between 0 and 1 ).

A bit change process in the BPSO is: 
bin string $= \begin{cases}1, & X_{i d} \geq 0.5 \\ 0, & X_{i d}<0.5\end{cases}$

$\mathrm{X}_{\mathrm{id}}$ has been transmitted for polynomial structure selection in BPSO for each one particle. The forecasting of the parameter value, $\theta_{\mathrm{R}}$ utilizes $\mathrm{QR}$ factorization.

$\mathrm{P}_{\mathrm{R}} \theta_{\mathrm{R}}+\varepsilon=\mathrm{y}$

$P_{R}=Q_{R} R_{R}$

$\mathrm{g}_{\mathrm{R}}=\mathrm{Q}_{\mathrm{R}}^{\mathrm{T}} \mathrm{y}$

$\mathrm{R}_{\mathrm{R}} \theta_{\mathrm{R}}=\mathrm{g}_{\mathrm{R}}$

Then, reorganizing and solving Equation (10) leads to prediction of $\theta_{\mathrm{R}}$.

$\theta_{\mathrm{R}}=\mathrm{R}_{\mathrm{R}}^{\mathrm{T}} \mathrm{g}_{\mathrm{R}}$

\subsection{Model Estimation}

The residuals Normalized Sum Squared Error (NSSE), $V_{N S S E}\left(\theta, Z^{N}\right)$ based on the model parameters $(\theta)$ is:

$\mathrm{V}_{\mathrm{NSSE}}\left(\theta, \mathrm{Z}^{\mathrm{N}}\right)=\frac{1}{2 \mathrm{~N}} \sum_{\mathrm{t}=1}^{\mathrm{N}} \varepsilon^{2}(\mathrm{t}, \theta)$

The selection of the model order of AIC, FPE and MDL are shown in Equation (13), (14) and (15) respectively.

$\mathrm{V}_{\mathrm{AIC}}=\left(1+2 \frac{\mathrm{d}}{\mathrm{N}}\right) \mathrm{V}_{\mathrm{NSSE}}\left(\theta, \mathrm{Z}^{\mathrm{N}}\right)$

$\mathrm{V}_{\mathrm{MDL}}=\left(1+\log (\mathrm{N}) \frac{\mathrm{d}}{\mathrm{N}}\right) \mathrm{V}_{\mathrm{NSSE}}\left(\theta, \mathrm{Z}^{\mathrm{N}}\right)$

$\mathrm{V}_{\mathrm{FPE}}=\left(\frac{1+\frac{\mathrm{d}}{\mathrm{N}}}{1-\frac{\mathrm{d}}{\mathrm{N}}}\right) \mathrm{V}_{\mathrm{NSSE}}\left(\theta, \mathrm{Z}^{\mathrm{N}}\right)$

where $\mathrm{d}=$ number of estimated parameters and $\mathrm{N}=$ data points amount. The parsimony principle with the least model parameters amount is required for the valuable data fit besides the lowest $\mathrm{V}_{\mathrm{AIC}}, \mathrm{V}_{\mathrm{MDL}}$ and $\mathrm{V}_{\mathrm{FPE}}$.

The measured steam temperature data from SDPP is divided into both training and testing sets for model estimation and model validation respectively. The segmentation of the data for both sets is based on interlacing technique that divides the dataset based on the position. Training and testing set contains even and odd positions data respectively.

\subsection{Model Validation}

A One-Step Ahead (OSA) prediction is: 
$\hat{y}=\hat{g}(z(t))$

The regressors, $z(t)$ and estimated nonlinear model, $\hat{g}$ are utilized in OSA. $z(t)$ representation for the NARX model is:

$z(t)=\left[\begin{array}{c}y(t-1), y(t-2), \ldots \ldots, y\left(t-n_{y}\right), \\ u\left(t-n_{k}-1\right), u\left(t-n_{k}-2\right), \ldots \ldots, u\left(t-n_{k}-n_{u}\right)\end{array}\right]$

The Sum Squared Error (SSE) and Mean Squared Error (MSE) are employed for residuals magnitude testing and model fitting problems of the regression. The SSE and MSE equations for a residual vector $\varepsilon$ are:

$\operatorname{SSE}=\sum_{\mathrm{i}=1}^{\mathrm{n}}\left(\mathrm{e}_{\mathrm{i}}\right)^{2}=\sum_{\mathrm{i}=1}^{\mathrm{n}}\left(\mathrm{y}_{\mathrm{i}}-\hat{\mathrm{y}}_{\mathrm{i}}\right)^{2}$ (18) $\operatorname{MSE}=$

$\frac{\sum_{\mathrm{i}=1}^{\mathrm{n}}\left(\mathrm{e}_{\mathrm{i}}\right)^{2}}{\mathrm{n}}=\frac{\sum_{\mathrm{i}=1}^{\mathrm{n}}\left(\mathrm{y}_{\mathrm{i}}-\widehat{\mathrm{y}}_{\mathrm{i}}\right)^{2}}{\mathrm{n}}$

where $y_{i}=$ measured value, $\hat{y}_{i}=$ predicted value and $n=$ number of samples.

A low SSE and MSE values from the residuals magnitude indicates a good model fit. The performance of fit model is measured using R-Squared technique:

$\mathrm{R}^{2}=1-\frac{\sum_{\mathrm{i}=1}^{\mathrm{n}}\left(\mathrm{y}_{\mathrm{i}}-\widehat{\mathrm{y}}_{\mathrm{i}}\right)^{2}}{\sum_{\mathrm{i}=1}^{\mathrm{n}}\left(\mathrm{y}_{\mathrm{i}}-\overline{\mathrm{y}}_{\mathrm{i}}\right)^{2}}$

where $\bar{y}_{i}$ is mean value of $y$.

The nonlinear model applies cross correlation tests for the fitness exhaustive test. The existing data length finite amount in correlation tests aim $95 \%$ confidence limits. The residuals' whiteness model validity for the nonlinear model is executed using the following correlation tests.

$\theta_{\mathrm{uz}^{2}}(\tau)=\mathrm{E}\left[\mathrm{u}(\mathrm{t}-\tau) \varepsilon^{2}(\mathrm{t})\right]=0, \forall \tau$

$\theta_{\mathrm{u}^{\prime 2} \varepsilon}(\tau)=\mathrm{E}\left[\left(\mathrm{u}^{2}(\mathrm{t}-\tau)-\overline{\mathrm{u}}^{2}(\tau)\right) \varepsilon(\mathrm{t})\right]=0, \forall \tau$

$\theta_{\mathrm{u}^{\prime 2} \varepsilon^{2}}(\tau)=\mathrm{E}\left[\left(\mathrm{u}^{2}(\mathrm{t}-\tau)-\overline{\mathrm{u}}^{2}(\tau)\right) \varepsilon^{2}(\mathrm{t})\right]=0, \forall \tau$

where $\theta_{\mathrm{x}_{1} \mathrm{x}_{2}}(\tau)=$ correlation coefficient between signals $\mathrm{x}_{1}$ and $\mathrm{x}_{2}, \mathrm{E}[\boldsymbol{m}]=$ correlation mathematical expectation, $\varepsilon(\mathrm{t})=$ model residuals $=\mathrm{y}(\mathrm{t})-\hat{\mathrm{y}}(\mathrm{t}), \tau=$ lag space and $\mathrm{u}(\mathrm{t})=$ observed input at time $\mathrm{t}$.

The histogram examination shows the residuals distribution which necessitates a white noise symmetric bell-shaped dispersion. The most frequency amounts are expected to narrow off equally at the end of the tails and to cluster in the central. 


\subsection{Experimental Design}

SDPP system uses an immersed coil-type heater in water for steam generation. The water and steam temperature are supervised by the application of two resistive temperature detectors (RTD) PT-100 in the column. A 1 to $5 \mathrm{~V}$ is utilized for the resistance sensors output conversion for selected temperature range. Submersion heating component transmits $1.5 \mathrm{~kW}$, $240 \mathrm{~V}$ and $50 \mathrm{~Hz}$ power signal modulation with specific sampling time. All the temperature data are integrated through Data Acquisition (DAQ) card with the MATLAB as illustrated in Fig. 1.

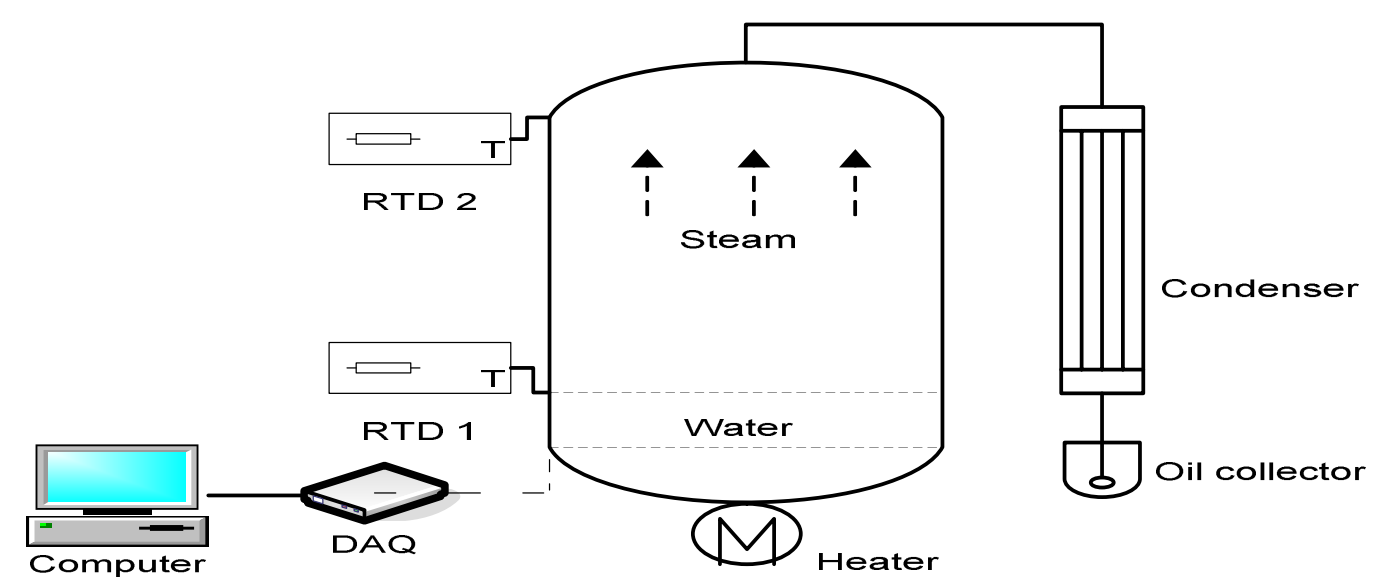

Fig.1. Steam distillation pilot plant (SDPP) system

Fig. 2 demonstrated 18, 000 of the nonlinear steam temperature dataset using PRBS input from the SDPP system.
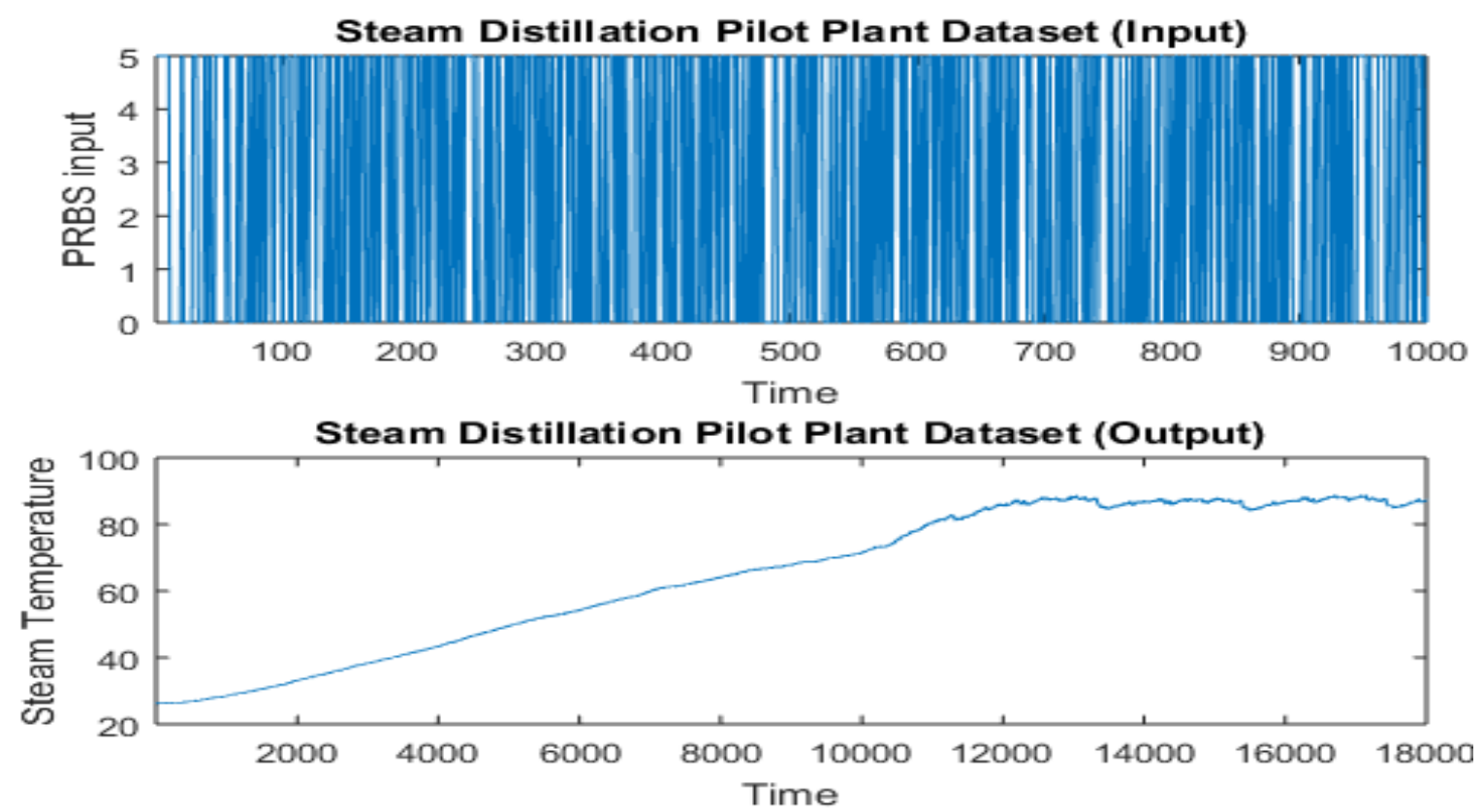

Fig.2. SDPP dataset 
Several maximum iterations, swarm sizes and random seeds parameters for optimization purposes have been tested. The maximum iterations discovery for each experiment leads to PSO search termination. Bigger potentiality in global minima searching is formed with greater swarm sizes based on computational rate and optimization period by the number of agents. Besides that, the final optimization PSO result is determined by each $V_{\text {id }}$ and $X_{i d}$ particles of the preliminary random seed. Dissimilar random seeds for repetitive tests decides the results consistency. Thus, the selected parameter values as in Table 1 for the fitness function optimal convergence searching are tested as been executed by [25, 27].

Table 1. BPSO parameter settings for structure selection

\begin{tabular}{cc}
\hline Parameter & Value \\
\hline Fitness Criterion & AIC, FPE, MDL \\
Swarm size & $10,20,30,40,50$ \\
Maximum Iterations & $500,1000,1500$ \\
Initial Random Seed & $0,10000,20000$ \\
$\mathrm{X}_{\min }$ & 0 \\
$\mathrm{X}_{\max }$ & 1 \\
$\mathrm{~V}_{\min }$ & -1 \\
$\mathrm{~V}_{\max }$ & +1 \\
$\mathrm{C}_{1}$ & 2.0 \\
$\mathrm{C}_{2}$ & 2.0 \\
\hline
\end{tabular}

\section{RESULTS AND DISCUSSION}

As presented in Table 2, the models were compared based on the model's selection criteria as in Equation (13)-(15). High R-squared and low MSE values have been reported by all the criterion designated to the acceptable fitting results. Not only that, the small number of CRV was presented for the residuals of all criterion. Consequently, the FPE model was found to be slightly better compared to AIC and MDL models because of the lowest fitness values and the least CRV on the testing set. 
Table 2. Model validation summary

\begin{tabular}{|c|c|c|c|}
\hline Fitness Criterion & Evaluation Criterion & Training Set & Testing Set \\
\hline \multirow{7}{*}{ AIC } & Times Found & \multicolumn{2}{|c|}{3} \\
\hline & AIC & $8.0429 \times 10^{-3}$ & $8.1621 \times 10^{-3}$ \\
\hline & FPE & $8.3150 \times 10^{-3}$ & $8.4383 \times 10^{-3}$ \\
\hline & MDL & $7.7357 \times 10^{-3}$ & $7.8503 \times 10^{-3}$ \\
\hline & R-squared (\%) & 99.9972 & 99.9973 \\
\hline & CRV & 22 & 23 \\
\hline & MSE & $1.2639 \times 10^{-2}$ & $1.2826 \times 10^{-2}$ \\
\hline \multirow{7}{*}{ FPE } & Times Found & \multicolumn{2}{|c|}{2} \\
\hline & AIC & $8.4391 \times 10^{-3}$ & $8.6166 \times 10^{-3}$ \\
\hline & FPE & $8.7246 \times 10^{-3}$ & $8.9081 \times 10^{-3}$ \\
\hline & MDL & $8.1167 \times 10^{-3}$ & $8.2874 \times 10^{-3}$ \\
\hline & R-squared (\%) & 99.9970 & 99.9970 \\
\hline & $\mathrm{CRV}$ & 22 & 20 \\
\hline & MSE & $1.3261 \times 10^{-2}$ & $1.3540 \times 10^{-2}$ \\
\hline \multirow{7}{*}{ MDL } & Times Found & \multicolumn{2}{|c|}{3} \\
\hline & AIC & $8.0429 \times 10^{-3}$ & $8.1621 \times 10^{-3}$ \\
\hline & FPE & $8.3151 \times 10^{-3}$ & $8.4385 \times 10^{-3}$ \\
\hline & MDL & $7.7357 \times 10^{-3}$ & $7.8505 \times 10^{-3}$ \\
\hline & R-squared (\%) & 99.9972 & 99.9971 \\
\hline & CRV & 22 & 23 \\
\hline & MSE & $1.2639 \times 10^{-2}$ & $1.2827 \times 10^{-2}$ \\
\hline
\end{tabular}

In addition, a minimum number of parameters as in Table 3 was also obtained for all models' criterion. In this search, the NARX-based BPSO model managed to cut down the CRV numbers, maintained low fitness value and the smallest quantity of parameters. The steam temperature NARX-based BPSO models for AIC, FPE and MDL models are reported next. 
Table 3. Output model

\begin{tabular}{|c|c|}
\hline Criterion & Output Model \\
\hline \multirow{4}{*}{ AIC } & $\mathrm{y}(\mathrm{t})=1.0011 \mathrm{y}(\mathrm{t}-4)+4.9199 \times 10^{-5} \mathrm{u}(\mathrm{t}-4) * \mathrm{y}(\mathrm{t}-2)$ \\
\hline & $-1.1082 \times 10^{-2} \quad y(t-4) * y(t-4)+1.0624 \times 10^{-3} \quad y(t-2) * y(t-4)$ \\
\hline & $+2.5963 \times 10^{-3} y(t-2) * y(t-3)$ \\
\hline & $+7.4095 \times 10^{-3} y(t-1)^{*} y(t-4)+\varepsilon(t)$ \\
\hline \multirow{4}{*}{ FPE } & $\mathrm{y}(\mathrm{t})=1.0011 \mathrm{y}(\mathrm{t}-4)+4.9238 \times 10^{-5} \mathrm{u}(\mathrm{t}-4) * \mathrm{y}(\mathrm{t}-2)-2.1099 \times 10^{-2} \mathrm{y}(\mathrm{t}-2) * \mathrm{y}(\mathrm{t}-4)$ \\
\hline & $+2.5967 \times 10^{-3} y(t-2)^{*} y(t-3)$ \\
\hline & $+7.4104 \times 10^{-3} y(t-1)^{*} \mathrm{y}(\mathrm{t}-4)$ \\
\hline & $+1.1078 \times 10^{-2} y(t-2) * y(t-2)+\varepsilon(t)$ \\
\hline \multirow{4}{*}{ MDL } & $y(t)=1.0007 y(t-2)-1.7632 \times 10^{-3} u(t-2)$ \\
\hline & $+4.7610 \times 10^{-4} u(t-1)^{*} u(t-2)+5.5021 \times 10^{-5} \quad u(t-4)^{*} y(t-3)$ \\
\hline & $-8.6475 \times 10^{-3} y(t-2) * y(t-4)$ \\
\hline & $+8.6386 \times 10^{-3} y(t-1)^{*} y(t-4)+\varepsilon(t)$ \\
\hline
\end{tabular}

Based on Table 3, the output models selected using different criteria were reported. Consequently, all the three models were found to be effective with the minimum number of parameters of the output model. The parsimonious model structures are an indication of good model fit as the minimum parameter in the output model were necessary.

The BPSO-based NARX justification results representing the FPE model are presented. Fig. 3 shows good model resulted by high R-squared. Additionally, small MSE value is demonstrated in Fig. 4. The FPE model recorded little violations in the correlation plots (Fig. 5 until Fig. 7) and well distributed of the white noise in histogram tests (Fig. 8).

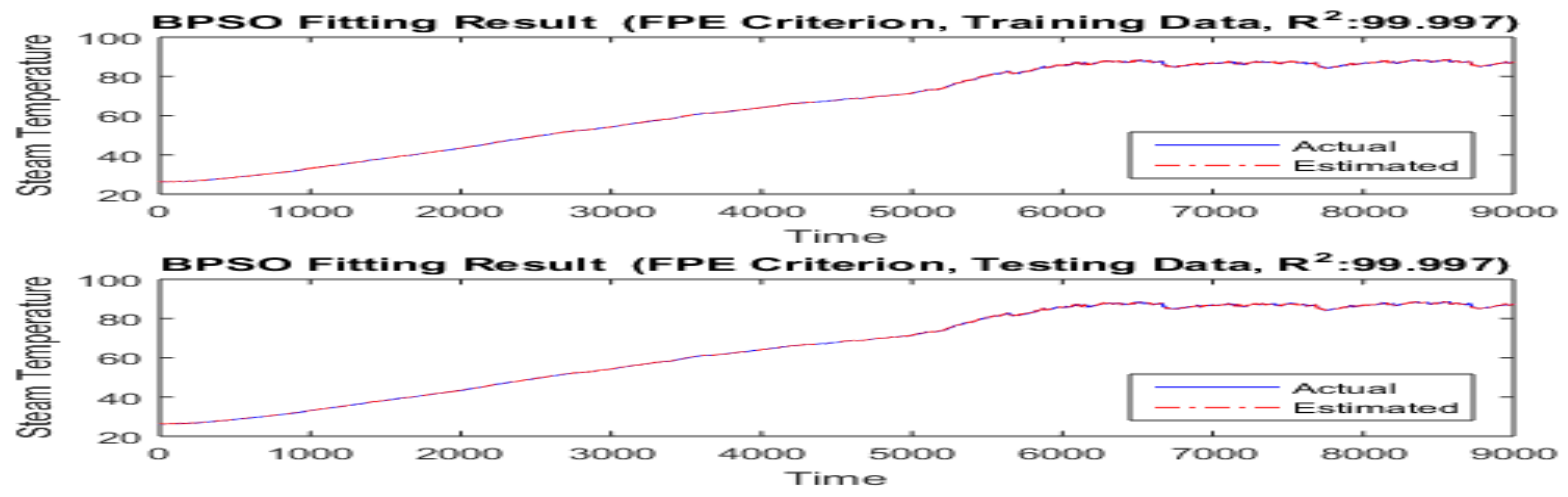

Fig.3. BPSO steam temperature model fit 


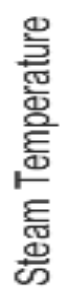

BPSO Residuals (FPE Criterion, Training Data, MSE:0.013261)

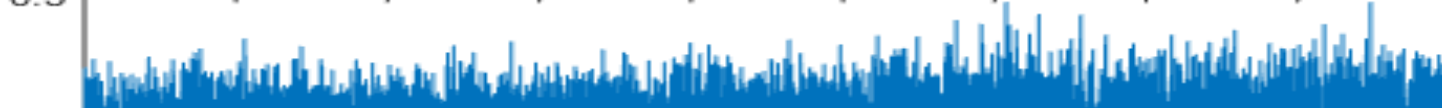

o

$-0.5$

Hinn

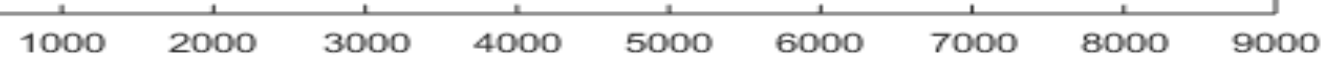

Time

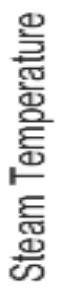

BPSO Residuals (FPE Criterion, Testing Data, MSE:0.01354)

-0.5

Fig.4. BPSO steam temperature residual plot

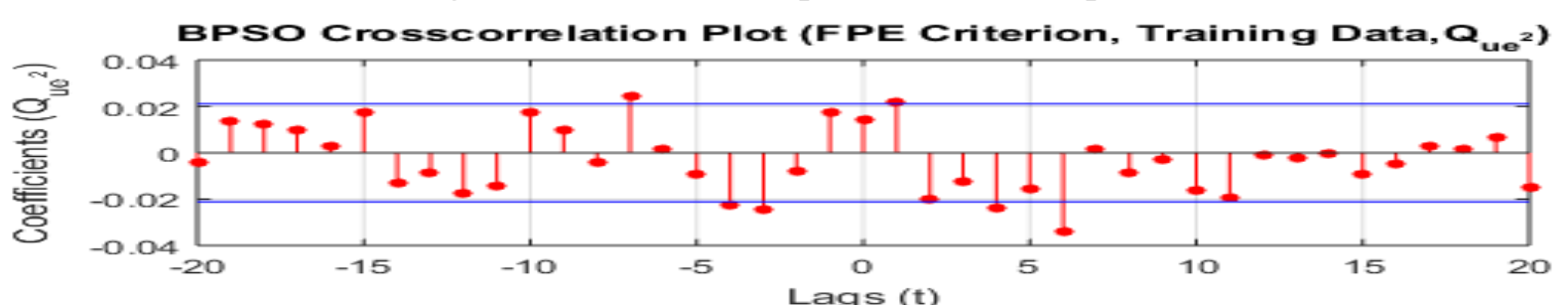

Lags (t)

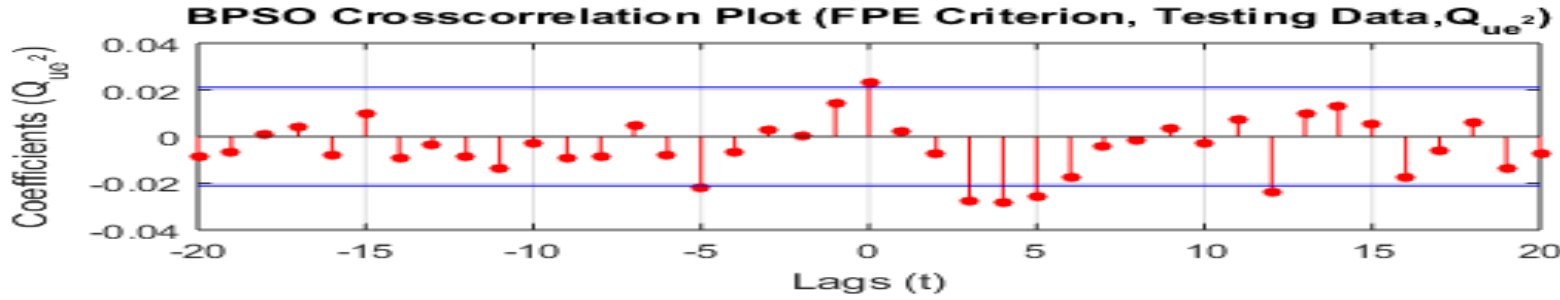

Fig.5. BPSO steam temperature correlation test (1/3)

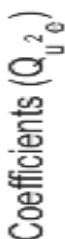

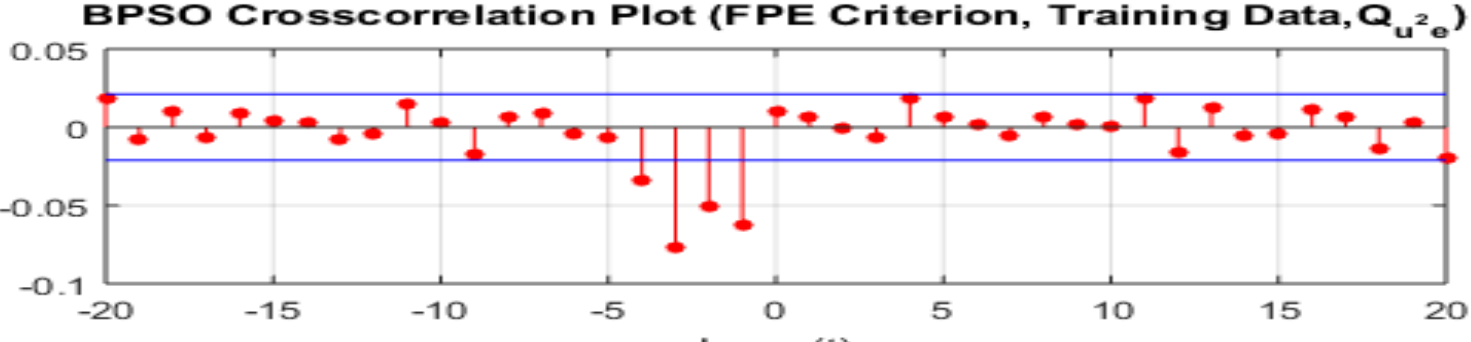

Lags ( $t$ )

BPSO Crosscorrelation Plot (FPE Criterion, Testing Data, $\mathbf{Q}_{\mathbf{u}^{2}}{ }_{\mathrm{e}}$ )

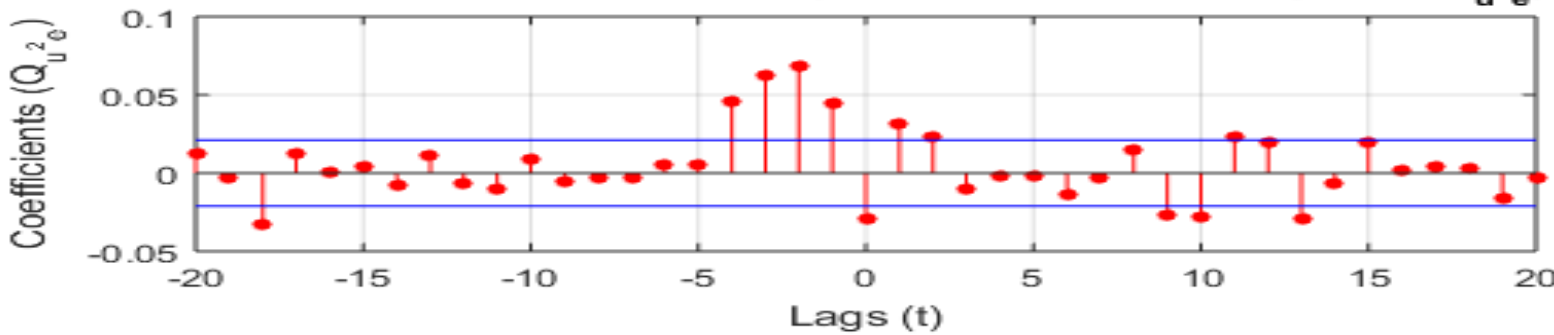

Fig.6. BPSO steam temperature correlation test $(2 / 3)$ 

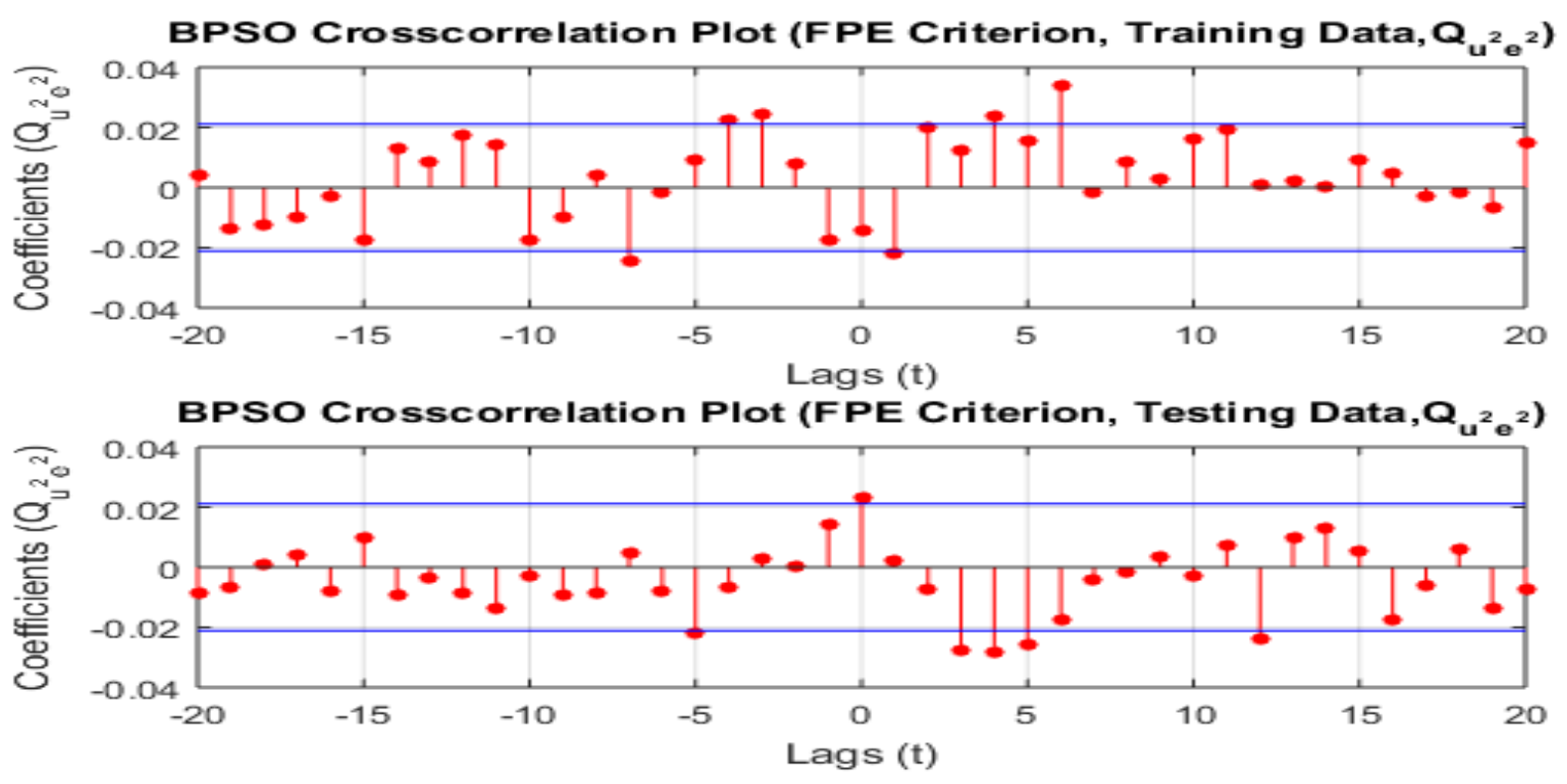

Fig.7. BPSO steam temperature correlation test (3/3)
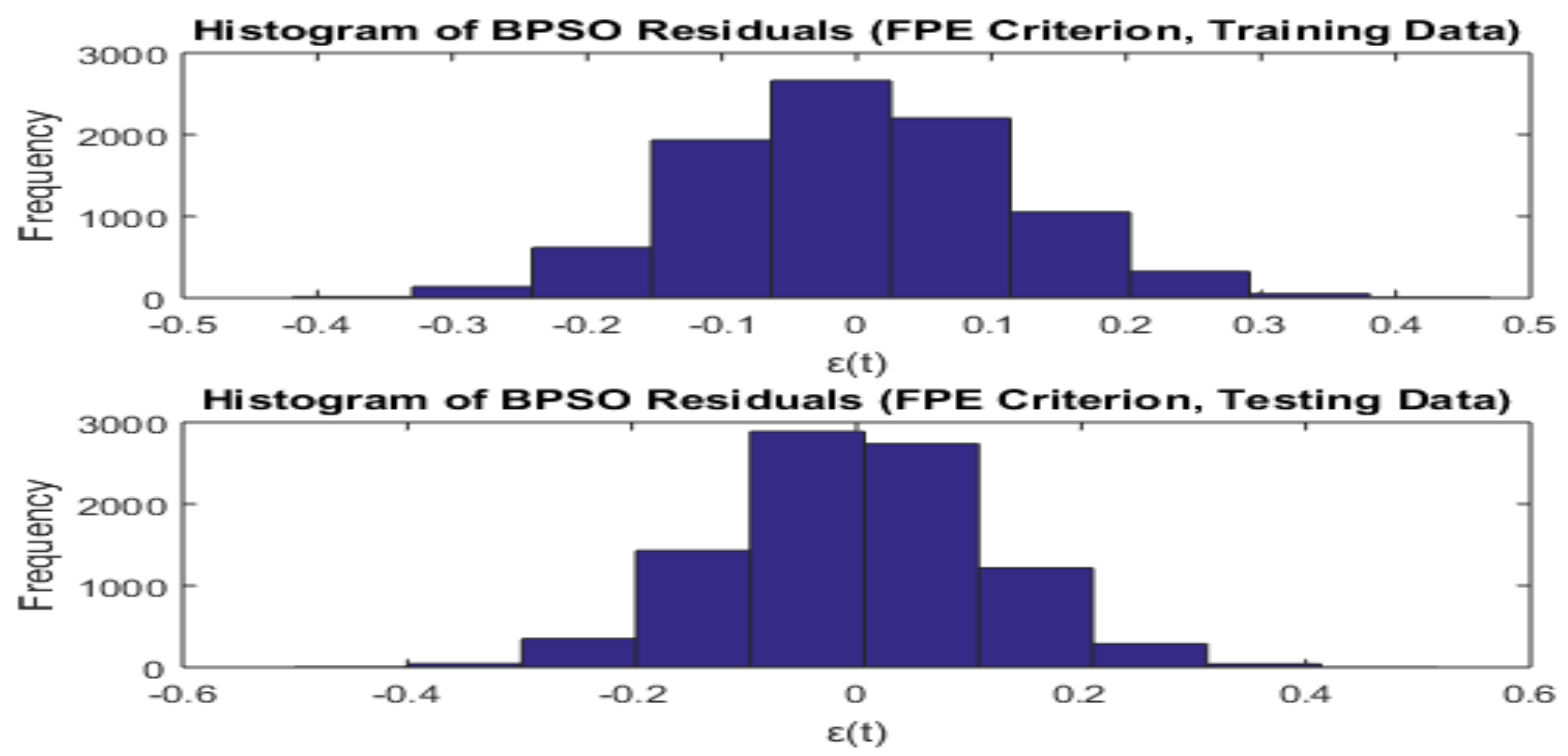

Fig.8. BPSO steam temperature histogram of residuals

Thus, the FPE model's residual displayed small correlation as indicated within $95 \%$ confidence limit in the correlation plots with low deviations number. Therefore, all criterion models generated for BPSO-based NARX were measured efficient and exact illustrations of the nonlinear system.

The parameters adjustment for BPSO as in Table 1 was investigated. Fig. 9 reported that swarm size 20 showed the lowest fitness values for FPE compared to other swarm sizes and model criterion. Subsequently, the insignificant outcome on the fitness for the maximum iterations parameter was indicated in Fig. 10. As well as a small number of iterations in Fig. 
11 which is lesser than 14 for convergence of iteration was obtained. In addition, few random seed values in Fig. 12 produced similar average fitness values with FPE recorded slightly better fitness compared to AIC and MDL.

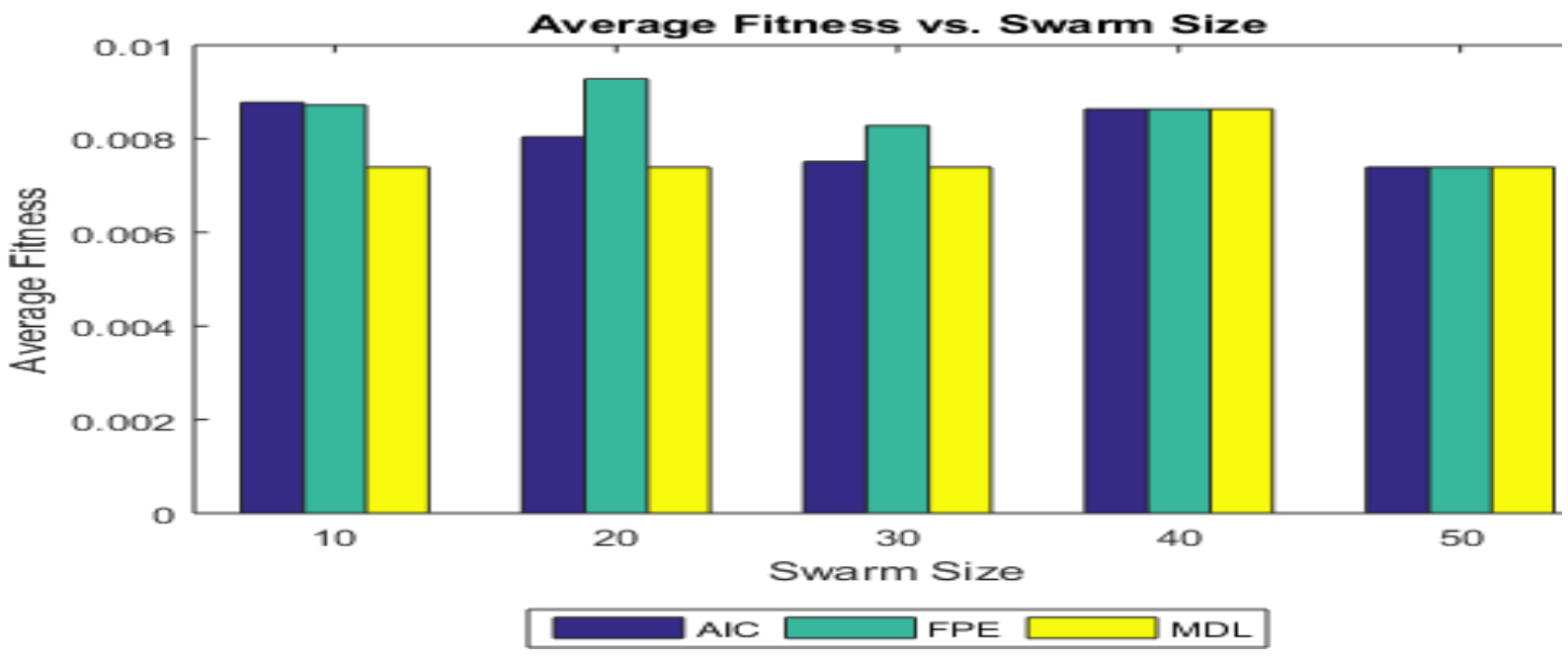

Fig.9. Average fitness vs. swarm size

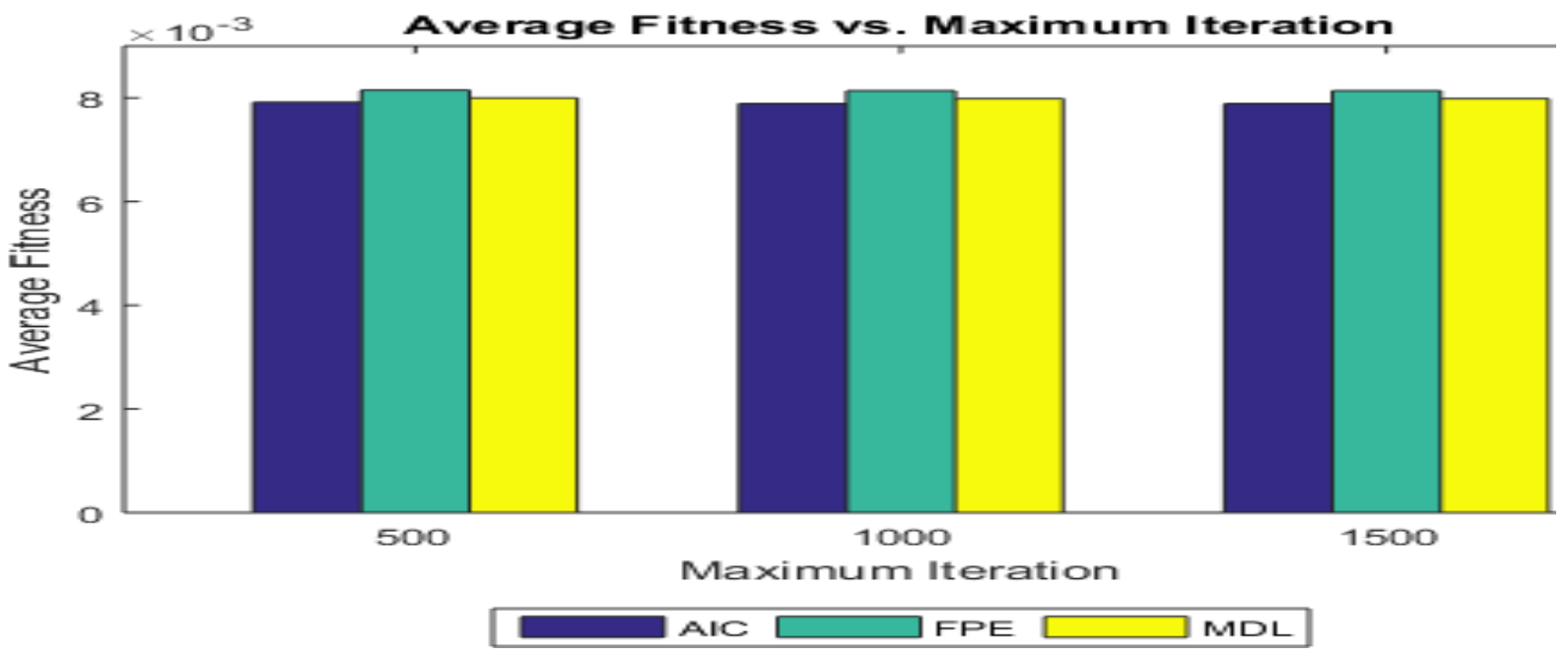

Fig.10. Average fitness vs. maximum iteration

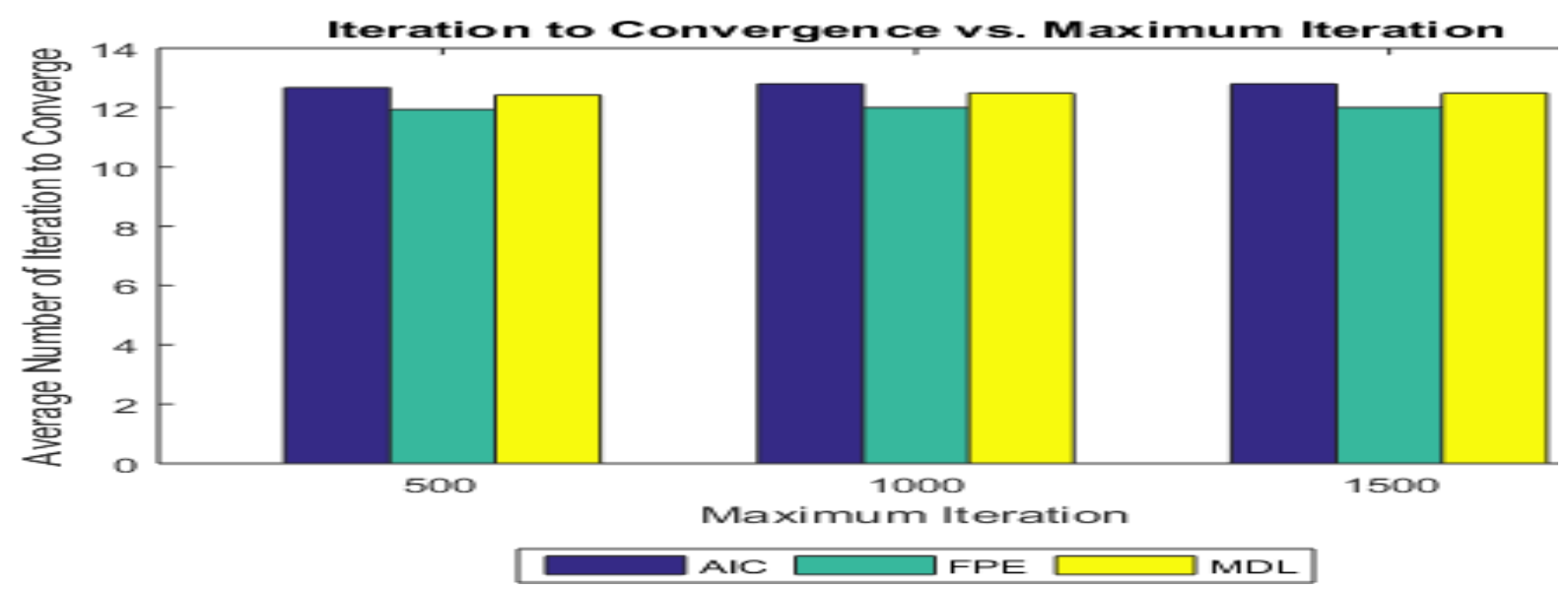

Fig.11. Iteration to convergence vs. maximum iteration 


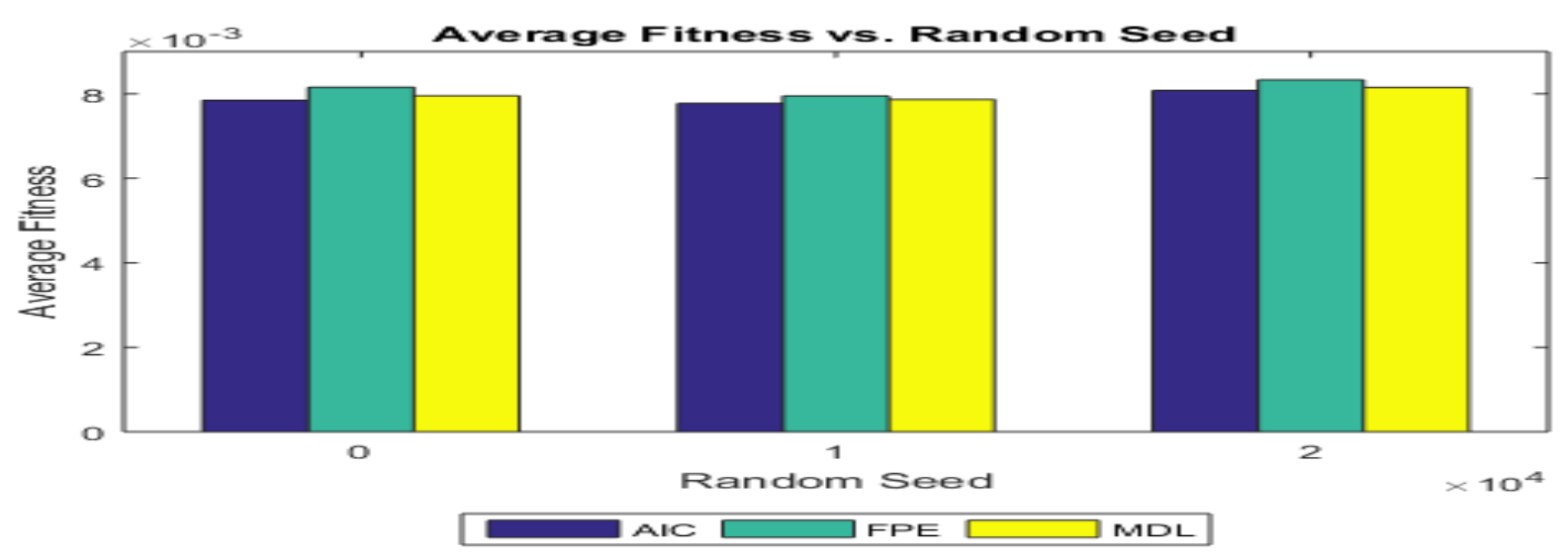

Fig.12. Average fitness vs. random seed

\section{CONCLUSION}

The nonlinear modelling for steam temperature using NARX-based BPSO algorithm structure was presented for the SDPP identification. The effects of BPSO parameter adjustment on the fitness was also identified. The nonlinear models recorded not only better combination due to its low total CRV from both training and testing sets, and small fitness value but also obtained lesser parameter number of the output model. Subsequently, the FPE model was observed to be a slightly greater model because it had low fitness values, besides the least CRV on the testing set compared to AIC and MDL. Subsequently, for the parameter adjustment, swarm size 20 showed the lowest fitness values for FPE. Another essential point, there is no significant outcome with the maximum iterations and random seed modification on the fitness. Overall, all criterion models generated for NARX-based BPSO were considered valid and accurate representations of the system with FPE model presented as a slightly better model [29]. This optimization of the nonlinear modelling is encouraging and suggest the opportunities for future research with another type of perturbation input signals for instance, Multi-level Pseudo Random Sequence (M-level PRS), Multi-Sine (M-Sine) and Random Gaussian Signal (RGS).

\section{ACKNOWLEDGEMENTS}

The author would like to thank SLAB/SLAI Ministry of Higher Education Malaysia for their scholarship. The authors would like to graciously acknowledge the Ministry of Higher Education and Universiti Teknologi MARA for supporting this research work through Grant 
No: 600-IRMI/MYRA 5/3/LESTARI(0040/2016).

\section{REFERENCES}

[1] Billings S A, Chen S, Backhouse R J. The identification of linear and non-linear models of a turbocharged automotive diesel engine. Mechanical Systems and Signal Processing, 1989, 3(2):123-142

[2] Hanafi D, Suid M S, Ribuan M N, Omar R O, Than M N, Rahmat M F. Speed effect to a quarter car ARX model based on system identification. International Journal on Advanced Science, Engineering and Information Technology, 2017, 7(2):468-474

[3] Guo Y, Guo L Z, Billings S A, Wei H L. Identification of nonlinear systems with non-persistent excitation using an iterative forward orthogonal least squares regression algorithm. International Journal of Modelling, Identification and Control, 2015, 23(1):1-7

[4] Aggoune L, Chetouani Y, Radjeai H. Change detection in a distillation column using non-linear auto-regressive moving average with exogenous input model and Hellinger distance. IET Science, Measurement and Technology, 2016, 10(1):10-17

[5] Yang Y, Barton P I. Refinery optimization integrated with a nonlinear crude distillation unit model. IFAC-PapersOnLine, 2015, 48(8):205-210

[6] Cong L, Chang L, Liu X. Nonlinear-wave based analysis and modeling of heat integrated distillation column. Separation and Purification Technology, 2015, 150:119-131

[7] Cong L. Nonlinear wave-based measurement location design and online estimation of a high-purity heat-integrated distillation column. Chemical Engineering and Technology, 2016, 39(12):2196-2206

[8] Harrou F, Madakyaru M, Sun Y. Improved nonlinear fault detection strategy based on the Hellinger distance metric: Plug flow reactor monitoring. Energy and Buildings, 2017, 143:149-161

[9] Paduart J, Lauwers L, Swevers J, Smolders K, Schoukens J, Pintelon R. Identification of nonlinear systems using polynomial nonlinear state space models. Automatica, 2010, 46(4):647-656

[10] Shariff H M, Marzaki M H, Tajjudin M, Rahiman M H. System identification for steam distillation pilot plant: Comparison between linear and nonlinear models. In IEEE 3rd International Conference on System Engineering and Technology, 2013, pp. 263-268 
[11] Araromi D O, Sonibare J A, Emuoyibofarhe J O. Fuzzy identification of reactive distillation for acetic acid recovery from waste water. Journal of Environmental Chemical Engineering, 2014, 2(3):1394-1403

[12] Kougioumtzoglou I A, Spanos P D. An identification approach for linear and nonlinear time-variant structural systems via harmonic wavelets. Mechanical Systems and Signal Processing, 2013, 37(1):338-352

[13] Jacobs W R, Wilson E D, Assaf T, Rossiter J, Dodd T J, Porrill J, Anderson S R. Control-focused, nonlinear and time-varying modelling of dielectric elastomer actuators with frequency response analysis. Smart Materials and Structures, 2015, 24(5):1-18

[14] Li Y, Wei H L, Billings S A, Sarrigiannis P G. Identification of nonlinear time-varying systems using an online sliding-window and common model structure selection (CMSS) approach with applications to EEG. International Journal of Systems Science, 2016, 47(11):2671-2681

[15] Kiefer J M, Johnson M D, Wall J H, Dominguez A. Applied time domain stability margin assessment for nonlinear time-varying systems. In 227th American Astronomical Society, 2016, pp. 1-2

[16] Kasuan N, Ismail N, Taib M N, Rahiman M H. Recurrent adaptive neuro-fuzzy inference system for steam temperature estimation in distillation of essential oil extraction process. In IEEE 7th International Colloquium on Signal Processing and its Applications, 2011, pp. 1-6

[17] Kasuan N, Taib M N, Rahiman M H. Model reference adaptive controller to regulate steam temperature in distillation process for essential oil extraction. In IEEE 7th International Colloquium on Signal Processing and its Applications, 2011 pp. 298-303

[18] Yazid E, Liew M S, Parman S, Kurian V J. Identification of transfer functions from surge motion response of a semisubmersible platform using time-varying NARX model. Applied Ocean Research, 2016, 54:53-66

[19] Billings S A, Wei H L. An adaptive orthogonal search algorithm for model subset selection and non-linear system identification. International Journal of Control, 2008, 81(5):714-724

[20] Mendez E M, Billings S A. An alternative solution to the model structure selection problem. IEEE Transactions on Systems, Man, and Cybernetics-PART A: Systems and Humans, 2001, 31(6):597-608 
[21] Mohd Yassin A I. Nonlinear auto-regressive model structure selection using binary particle swarm optimization algorithm. PhD thesis, Selangor: Universiti Teknologi MARA, 2014

[22] Keshavarzi R, Akhlaghi M, Emami F. Binary PSO algorithm assisted to investigate the optical sensor based plasmonic nano-bi-domes. Optik-International Journal for Light and Electron Optics, 2016, 127(19):7670-7675

[23] Haixiang G, Yijing L, Yanan L, Xiao L, Jinling L. BPSO-Adaboost-KNN ensemble learning algorithm for multi-class imbalanced data classification. Engineering Applications of Artificial Intelligence, 2016, 49:176-193

[24] Yassin I M, Zabidi A, Ali M S, Tahir N M, Abidin H Z, Rizman Z I. Binary particle swarm optimization structure selection of nonlinear autoregressive moving average with exogenous inputs (NARMAX) model of a flexible robot arm. International Journal on Advanced Science, Engineering and Information Technology, 2016, 6(5):630-637

[25] Yassin I M, Taib M N, Adnan R, Salleh M K, Hamzah M K. Effect of swarm size parameter on Binary Particle Swarm optimization-based NARX structure selection. In IEEE Symposium on Industrial Electronics and Applications, 2012, pp. 219-223

[26] Awadz F, Yassin I M, Rahiman M H, Taib M N, Zabidi A, Hassan H A. System identification of essential oil extraction system using Non-Linear Autoregressive Model with Exogenous Inputs (NARX). In IEEE Control and System Graduate Research Colloquium. 2010, pp. 20-25

[27] Yassin I M, Taib M N, Adnan R. Extended analysis of BPSO structure selection of nonlinear auto-regressive model with exogenous inputs (NARX) of direct current motor. Songklanakarin Journal of Science and Technology, 2014, 36(6):683-699

[28] Zabidi A, Tahir N M, Yassin I M, Rizman Z I. The performance of binary artificial bee colony (BABC) in structure selection of polynomial NARX and NARMAX models. International Journal on Advanced Science, Engineering and Information Technology, 2017, $7(2): 373-379$

[29] Yassin I M, Jailani R, Ali M, Baharom R, Hassan A, Rizman Z I. Comparison between cascade forward and multi-layer perceptron neural networks for NARX functional Electrical Stimulation (FES)-based muscle model. International Journal on Advanced Science, Engineering and Information Technology, 2017, 7(1):215-221. 


\section{How to cite this article:}

Hambali N, Taib MN, Yassin AIM, Rahiman MHF. Narx-based bpso modelling for time-varying steam temperature of steam distillation pilot plant. J. Fundam. Appl. Sci., 2017, 9(4S), 94-110. 